\title{
Notes on Modular Conjugations \\ of von Neumann Factors
}

\author{
M. Leitz-Martini and M. Wollenberg
}

\begin{abstract}
In this paper we present some results about the characterization of modular conjugations of von Neumann algebras. Further, we show that hyperfinite factors of type II, $\mathrm{III}_{1}$, and $I I I_{\lambda}$ have algebraic conjugations which are not modular conjugations.
\end{abstract}

Keywords: Operator algebras, modular theory, modular conjugation

AMS subject classification: 46 L 10

\section{Introduction}

Let $\mathcal{M}$ be a von Neumann algebra on a separable Hilbert space $\mathcal{H}$ and let $u$ be a cyclic and separating vector for $\mathcal{M}$. Then the Tomita operator $S$, the modular operator $\Delta$, and the modular conjugation $J$ are defined by

$$
S_{o} M u=M^{*} u, M \in \mathcal{M}, \quad S=\bar{S}_{o}, \quad \Delta=S^{*} S, \quad S=J \Delta^{\frac{1}{2}} .
$$

These modular objects $(\Delta, J)$ for $(\mathcal{M}, u)$ satisfy the following relations (see, e.g., [3, 14]):

$$
\begin{aligned}
J^{2} & =1 \text { and } J \text { is antiunitary } \\
J \Delta J & =\Delta^{-1} \\
J u & =u \\
\Delta u & =u \\
J \mathcal{M} J & =\mathcal{M}^{\prime} .
\end{aligned}
$$

Let $U$ be a unitary operator on $\mathcal{H}$. Then $v:=U u$ is cyclic and separating for $\mathcal{N}:=$ $U \mathcal{M} U^{*}$ and $(\mathcal{N}, v)$ has the modular objects

$$
\left(\Delta_{v}, J_{v}\right)=\left(U \Delta U^{*}, U J U^{*}\right)
$$

M. Leitz-Martini: Math. Inst. Univ., Auf der Morgenstelle 10, D-72076 Tübingen

M. Wollenberg: Inst. Math. Univ., Augustusplatz 10/11, D-04109 Leipzig

e-mail: martin.leitz@uni-tuebingen.de and wollenbg@mathematik.uni-leipzig.de

ISSN 0232-2064 / \$2.50 (C) Heldermann Verlag Berlin 
We call an operator $J$ satisfying (1) a conjugation. A conjugation $J$ satisfying (5) is called an algebraic conjugation for $\mathcal{M}$ (in [15: p. 337] it is called a unitary involution of $(\mathcal{M}, \mathcal{H}))$.

Now we are interested in the following questions:

(a) Let $J$ be a conjugation on a Hilbert space $\mathcal{H}$. Does there exist a von Neumann algebra $\mathcal{M}$ with a cyclic and separating vector $u$ such that $J$ is the modular conjugation for $(\mathcal{M}, u)$ ?

(b) Let $J$ be a conjugation on $\mathcal{H}$ and let $\Delta$ be the modular operator for $(\mathcal{M}, u)$ where $\mathcal{M}$ is a von Neumann algebra on $\mathcal{H}$ with the cyclic and separating vector $u$. Suppose (2) holds. Does there exist a von Neumann algebra $\mathcal{M}_{0}$ with a cyclic and separating vector $u_{o}$ such that $(\Delta, J)$ are the modular objects for $\left(\mathcal{M}_{o}, u_{o}\right)$ ?

(c) Let $J$ be an algebraic conjugation for the von Neumann algebra $\mathcal{M}$. Does there exist a cyclic and separating vector $u$ for $\mathcal{M}$ such that $J$ is even the modular conjugation for $(\mathcal{M}, u)$ ?

The answer to question (a) is yes (at least if the Hilbert space is infinite-dimensional) and very easy to show. For completeness we discuss this simple question in Section 2 . In Section 3 we show that question (b) can be answered affirmatively. The answer to question (c) is in general no. This question (and a partial answer without proof) is mentioned in [14: p. 321] in connection with a possible difference between standard von Neumann algebras and hyperstandard von Neumann algebras. We treat question (c) in some detail in Section 4.

The modular theory plays an important role in the theory of von Neumann algebras and in the algebraic approach to quantum physics (see, e.g., $[2,5,8]$ ). Modular conjugations are used for studying different problems in quantum field theory (see, e.g., $[2,8$, 12]). In particular, the characterizations of modular conjugations, which we investigate here, are - for example - important for some inverse problems of the modular operator (see, e.g., $[4,12,17]$ ).

In this paper we only consider von Neumann algebras which are factors, shortly von Neumann factors, and which are separable, i.e. they can be represented faithfully on a separable Hilbert spacc.

If $\mathcal{M}$ is a von Neumann factor acting on a separable Hilbert space $\mathcal{H}$, then we denote by $M C(\mathcal{M})$ the set of modular conjugations for $\mathcal{M}$. This means that $J \in M C(\mathcal{M})$ if and only if there is a cyclic and separating vector $u$ for $\mathcal{M}$ such that $J$ is the modular conjugation for $(\mathcal{M}, u)$.

We will use the notation unit vector for a vector with norm 1 and antiunitary operator for an operator $V$ which is antilinear, i.e. $V(c u)=\bar{c} V u$, and satisfies $V V^{*}=$ $V^{*} V=1$. Further, we denote the set of unitaries of $\mathcal{M}$ by $\mathcal{U}(\mathcal{M})$. 


\section{Conjugations and modular conjugations}

First we are interested in the question whether a conjugation is always a modular conjugation for some von Neumann factor with a cyclic and separating vector. The answer is known and we will present a simple proof. For this proof we use the following lemma about conjugations.

Lemma 2.1. Let $J_{1}$ and $J_{2}$ be two conjugations acting on a separable Hilbert space $\mathcal{H}_{\text {o. Then: }}$

(i) There is a unitary operator $U$ on $\mathcal{H}_{o}$ such that $J_{2}=U J_{1} U^{*}$ and the spectral projections of $U$ commute with both $J_{1}$ and $J_{2}$.

(ii) Let $A$ be a positive selfadjoint operator on $\mathcal{H}_{0}$ such that $J_{i} A J_{i}=A^{-1}(j=1,2)$. Then the unitary operator $U$ from (i) can be chosen such that additionally $U A U^{*}=A$.

Proof. 1. First we note that the relation $J W J=W^{*}$ for a conjugation $J$ and a unitary operator $W$ implies that $J$ commutes with the spectral projections of $W$, $J E_{W}(\Gamma) J=E_{W}(\Gamma)$ with $\Gamma \subset[0,2 \pi]$. This follows from the spectral representation $W=\int_{0}^{2 \pi} e^{i \mu} E_{W}(d \mu)$ (see, e.g., [1: p. 46]).

2. We set $V=J_{2} J_{1}$. The operator $V$ is unitary and $1=\left(J_{2}\right)^{2}=V J_{1} \cdot V J_{1}$ implies that $J_{1} V J_{1}=V^{*}$. Thus, by Step 1 , the spectral projections $E_{V}(\Gamma)$ commute with $J_{1}$. We set $U=\int_{0}^{2 \pi} e^{i \frac{\mu}{2}} E_{V}(d \mu)$. The functional calculus gives $V=U \cdot U$ (see, e.g., [1: p. 46]) and $J E_{V}(\Gamma)=E_{V}(\Gamma) J$ implies $J_{1} U J_{1}=U^{*}$. From $V J_{1}=J_{2}$ therefore $J_{2}=V J_{1}=U U J_{1}=U J_{1} U^{*}$ follows. Further,

$$
J_{2} U J_{2}=V J_{1} U V J_{1}=U U J_{1} U U U J_{1}=U U \cdot U^{*} U^{*} U^{*}=U^{*}
$$

because of $J_{1} U=U^{*} J_{1}$. The relations $J_{i} U J_{i}=U^{*}(i=1,2) \mathrm{imply}$, by Step 1 , that the spectral projections of $U$ commute with $J_{1}$ and $J_{2}$. This shows that $U$ satisfies all desired relations in (i).

3. From $J_{i} A J_{i}=A^{-1}(i=1,2)$ it follows, with $V=J_{2} J_{1}$, that

$$
V A V^{*}=J_{2} J_{1} A J_{1} J_{2}=J_{2} A^{-1} J_{2}=A \text {. }
$$

Thus the spectral projections of $V$ commute with $A$ and, by the definition of $U$ above, $U$ commutes with $A$, too

Now we come to the answer of the mentioned question.

Proposition 2.2. Let $\mathcal{H}_{0}$ be a separable Hilbert space with $\operatorname{dim} \mathcal{H}_{0}=m^{2}$ for a natural number $m$ or $\operatorname{dim} \mathcal{H}_{o}=\infty$. Let $K$ be a conjugation on $\mathcal{H}_{0}$. Then there is a von Neumann factor $\mathcal{M}_{0}$ on $\mathcal{H}_{0}$ with a cyclic and separating vector $u_{0}$ such that $K$ is the modular conjugation for $\left(\mathcal{M}_{o}, u_{0}\right)$, i.e. $K \in M C\left(\mathcal{M}_{o}\right)$.

Proof. 1. The assumption on the dimension of $\mathcal{H}_{0}$ secures that we can identify $\mathcal{H}_{0}$ with a tensor product $\mathcal{K} \bar{\otimes} \mathcal{K}$. We define $\mathcal{M}=\mathcal{L}(\mathcal{K}) \bar{\otimes} 1$ and $u=\sum_{i} \lambda_{i} e_{i} \otimes e_{i}$ where $\left(c_{i}\right)_{i}$ is a basis in $\mathcal{K}$ and $\lambda_{i}>0$ with $\sum_{i} \lambda_{i}^{2}=1$. It is easy to show that $u$ is a cyclic and separating vector for $\mathcal{M}$.

2. Now let $(\Delta, J)$ be the modular objects for $(\mathcal{M}, u)$. For the two conjugations $J$ and $K$ there exist a unitary operator $U$ such that $U J U^{*}=K$ (see Lemma 2.1). We put $u_{o}=U u_{\text {. and }} \mathcal{M}_{0}=U M U^{*}$. Then (6) implies that $u_{o}$ is a cyclic and separating vector for the von Neumann factor $\mathcal{M}_{o}$ and that the modular objects $\left(\Delta_{o}^{*}, J_{o}\right)$ for $\left(\mathcal{M}_{o}, \dot{u}_{o}\right)$ are given by $\Delta_{0}=U \Delta U^{*}$ and $J_{o}=U J U^{*}=K$. This concludes the proof 
This proposition says that the dimension requirement is sufficient to secure that a conjugation $K$ on $\mathcal{H}_{o}$ is a modular conjugation. It is also easy to see that the assumption on the dimension is necessary. Since without this assumption there is no von Neumann factor $\mathcal{M}$ on $\mathcal{H}_{o}$ with a cyclic and separating vector.

If we look at the proof of Proposition 2.2 we easily see that one can prove the following stronger result (in the case $\operatorname{dim} \mathcal{H}_{0}=\infty$ ):

Let $K$ be a conjugation on a separable infinite-dimensional Hilbert space. Let $\mathcal{N}$ be a separable von Neumann factor of type $I_{\infty}, I I$, or $I I I$. Then there is a von Neumann factor $\mathcal{M}_{0}$ on $\mathcal{H}_{0}$ such that $\mathcal{M}_{0}$ is isomorphic to $\mathcal{N}, \mathcal{M}_{0}$ has a cyclic and separating vector $u_{o}$, and $K$ is the modular conjugation for $\left(\mathcal{M}_{o}, u_{0}\right)$.

\section{Modular conjugations for a modular operator}

In this section we consider the question how the set of modular conjugations for a fixed modular operator looks like.

Proposition 3.1. Let $\left(\Delta_{o}, J_{o}\right)$ be the modular objects for $\left(\mathcal{M}_{o}, u_{o}\right)$. Let $J$ be a conjugation satisfying $J \Delta_{o}=\Delta_{o}^{-1} J$. Then there is a unitary operator $W$ such that $W \Delta_{0} W^{*}=\Delta_{o}, W J_{0} W^{*}=J, u:=W u_{0}$ is a cyclic and separating vector for $\mathcal{M}:=$ $W \mathcal{M}_{0} W^{*}$, and $\left(\Delta_{o}, J\right)$ are the modular objects for $(\mathcal{M}, u)$.

Proof. By Lemma 2.1 we have for the two conjugations $J$ and $J_{o}$ a unitary operator $W$ such that $W J_{o} W^{*}=J$ and $W \Delta_{0} W^{*}=\Delta_{o}$. Defining $\mathcal{M}=W \mathcal{M}_{0} W^{*}$ and $u=W u_{0}$ the proposition is proved with the help of (6)

Proposition 3.2. Let $\left(\Delta_{o}, J_{o}\right)$ be the modular objects for $\left(\mathcal{M}_{o}, u_{o}\right)$. Let $u$ be a unit eigenvector of $\Delta_{o}$ to the eigenvalue 1 . Let $J$ be a conjugation satisfying $J \Delta_{o}=\Delta_{o}^{-1} J$ and $J u=u$. Then there is a unitary operator $W$ such that $W \Delta_{o} W^{*}=\Delta_{o}, W J_{o} W^{*}=$ $J, u=W u_{o}, u$ is a cyclic and separating vector for $\mathcal{M}:=W \mathcal{M}_{0} W^{*}$, and $\left(\Delta_{o}, J\right)$ are the modular objects for $(\mathcal{M}, u)$.

Proof. 1. First we note that according to Proposition 3.1 there is a unitary $W_{1}$ such that $W_{1} \Delta_{o}=\Delta_{0} W_{1}, W_{1} J_{0} W_{1}^{*}=J, v:=W_{1} u_{0}$ is a cyclic and separating vector for $\mathcal{M}_{1}:=W_{1} \mathcal{M}_{0} W_{1}^{*}$, and $\left(\Delta_{o}, J\right)$ are the modular objects for $\left(\mathcal{M}_{1}, v\right)$.

2. We have $J v=v$ and $\Delta_{o} v=v$. According to Lemma 5.2 there is a unitary $V_{1}$ such that $V_{1} \Delta_{0}=\Delta_{0} V_{1}, V_{1} J V_{1}^{*}=J$ and $u=V_{1} v$. Then the pair $\left(V_{1} \mathcal{M}_{1} V_{1}^{*}, V_{1} v\right)$ has the modular objects $\left(V_{1} \Delta_{o} V_{1}^{*}, V_{1} J V_{1}^{*}\right)=\left(\Delta_{0}, J\right)$ because $\left(\mathcal{M}_{1}, v\right)^{\prime}$ has the modular objects $\left(\Delta_{o}, J\right)$. Setting $W=V_{1} W_{1}$ we find the desired result

From Propositions 3.1 and 3.2 we see first that the condition $J \Delta_{o}=\Delta_{o}^{-1} J$ is necessary and sufficient that a conjugation $J$ is a modular conjugation for a given modular operator $\Delta_{o}$. Second we see that each eigenvector $u$ for $\Delta_{o}$ and $J$ to the cigenvalue 1 is a cyclic and separating vector for some von Neumann factor $\mathcal{M}$ such that $\left(\Delta_{o}, J\right)$ are the modular objects for $(\mathcal{M}, u)$. Third we find that the construction of the von Neumann factor $\mathcal{M}$ is given by $\mathcal{M}=W \mathcal{M}_{0} W^{*}$ where $W$ depends only on $\triangle_{o}, J_{o}, J, u_{o}, u$ and does not depend on the whole algebra $\mathcal{M}_{o}$. 


\section{Algebraic conjugations and modular conjugations}

First we note a simple characterization of the set of modular conjugations for a von Neumann factor.

Proposition 4.1. Let $J_{0}$ be a modular conjugation for $\left(\mathcal{M}_{0}, u_{o}\right)$. Then

$$
M C\left(\mathcal{M}_{0}\right)=\left\{J=U^{*} J_{0} U: U \in \mathcal{U}\left(\mathcal{M}_{0}^{\prime}\right)\right\}
$$

Proof. 1. If $J$ is a modular conjugation for a pair $\left(\mathcal{M}_{o}, u_{0}\right)$, then $J=U^{*} J_{o} U$ with some $U \in \mathcal{U}\left(\mathcal{M}_{o}^{\prime}\right)$ (see, e.g., [14: p. 331]).

2. Conversely, let $J=U^{*} J_{0} U$ with some $U \in \mathcal{U}\left(\mathcal{M}_{o}^{\prime}\right)$. Then (6) gives that $J$ is the modular conjugation for $\left(U^{*} \mathcal{M}_{0} U, U^{*} u_{0}\right)$. But $U^{*} \mathcal{M}_{0} U=\mathcal{M}_{0}$ in our case. Thus we get $J \in M C\left(\mathcal{M}_{0}\right)$

Next we characterize the modular conjugations $J$ for $\mathcal{M}_{0}$ with the help of the unitary $V:=J J_{0}$.

Proposition 4.2. Let $\mathcal{M}_{o}$ be a von Neumann factor on a separable' Hilbert space $\mathcal{H}$ with a cyclic and separating vector $u_{o}$. Let $J_{o}$ be the modular conjugation for $\left(\mathcal{M}_{0}, u_{0}\right)$ and let $J$ be an algebraic conjugation for $\mathcal{M}_{o}$. Further let $V:=J J_{0}$. Then:

(i) ad $V \in$ aut $\mathcal{M}_{o}$.

(ii) ad $V \in$ int $\mathcal{M}_{0}$ if and only if $J \in M C\left(\mathcal{M}_{0}\right)$.

(iii) If int $\mathcal{M}_{o}=$ aut $\mathcal{M}_{o}$, then $J \in M C\left(\mathcal{M}_{0}\right)$.

Proof. (i). From (5) for $J$ and $J_{o}$ it follows that ad $V \in$ aut $\mathcal{M}_{o}$.

(ii) Now suppose $J$ is a modular conjugation. Then from Proposition 4.1 we get that $J=U^{*} J_{0} U$ for some $U \in \mathcal{U}\left(\mathcal{M}_{0}^{\prime}\right)$ and $V:=J J_{0}=U^{*} \cdot J_{0} U J_{0}$. Since $J_{0} U J_{0} \in \mathcal{U}\left(\mathcal{M}_{0}\right)$ and $U^{*} \in \mathcal{U}\left(\mathcal{M}_{o}^{\prime}\right)$ we find that ad $V$ is from int $\mathcal{M}_{0}$.

Conversely, suppose that ad $V$ is an inner automorphism of $\mathcal{M}_{0}$. Then we can decompose $V$ in the form $V=V_{1} \cdot V_{2}$ where $V_{1} \in \mathcal{U}\left(\mathcal{M}_{0}\right)$ and $V_{2} \in \mathcal{U}\left(\mathcal{M}_{0}^{\prime}\right)$. By $J^{2}=$ $V J_{o} V J_{o}=1$, we get $J_{o} V J_{o}=V^{*}$ and find $V^{*}=V_{2}^{*} V_{1}^{*}=J_{0} V_{1} V_{2} J_{o}=J_{0} V_{1} J_{0} \cdot J_{0} V_{2} J_{0}$ with $J_{o} V_{1} J_{o}, V_{2}^{*} \in \mathcal{M}_{o}^{\prime}$ and $J_{o} V_{2} J_{o}, V_{1}^{*} \in \mathcal{M}_{0}$. Now using the uniqueness of such a product from unitaries from $\mathcal{M}_{0}$ and $\mathcal{M}_{0}^{\prime}$ (see Lemma 5.1) we obtain $J_{0} V_{1} J_{0}=V_{2}^{*} e^{i c}$ and $J_{o} V_{2} J_{o}=V_{1}^{*} e^{-i c}$. By using the freedom in the decomposition $V_{1} \cdot V_{2}$ we can transform $c$ into zero. Therefore $V=J_{0} V_{2}^{*} J_{0} \cdot V_{2}=V_{2} \cdot J_{0} V_{2}^{*} J_{0}$ and $J=V J_{0}=$ $V_{2} J_{o} V_{2}^{*} J_{o} \cdot J_{o}=V_{2} J_{o} V_{2}^{*}$. This proves $J$ has the form of an element of $M C\left(\mathcal{M}_{0}\right)$ (see Proposition 4.1) and therefore $J$ is a modular conjugation. This proves the other direction of (ii).

(iii) It follows from (i) and (ii)

Remark 4.3. If we restrict ourselves to hyperfinite factors, then the property int $\mathcal{M}=$ aut $\mathcal{M}$ is only true for type I factors. At present it is not clear if there are (non-hyperfinite) type II factors with int $\mathcal{M}=$ aut $\mathcal{M}$. But we see from this proposition that every algebraic conjugation for a type I factor is even a modular conjugation for this factor.

From Proposition 4.2 we get a further characterization of algebraic conjugations. 
Proposition 4.4. Let $J_{o}, J, V$ be as in Proposition 4.2. Then:

(i) $J=V \cdot J_{0}=W \cdot J_{0} \cdot V$ with a unitary $W$ such that ad $W \in \operatorname{int} \mathcal{M}_{0}$.

(ii) $V^{2}=W, W^{*}=J_{o} \cdot W \cdot J_{o}$ and $V W V^{*}=W$.

Proof. (i) From the fact that ad $V$ is an automorphism of $\mathcal{M}_{0}$ and from (6) we get that $V J_{0} V^{*}$ is a modular conjugation for $\mathcal{M}_{0}$. Thus $V J_{0} V^{*}=W J_{0}$ with ad $W \in$ int $\mathcal{M}_{0}$ (see Proposition 4.2). This implies $J=V J_{0}=W J_{0} V$ where $W$ has the described properties. from

(ii) We get $V^{2}=W J_{0} V J_{0} \cdot V=W J_{0} V V^{*} J_{0}=W$. The second property follows

$$
J_{o} W J_{o}=J_{o} V^{2} J_{o}=J_{o}\left(J J_{o} \cdot J J_{o}\right) J_{o}=J_{o} J \cdot J_{o} J=V^{*} \cdot V^{*}=\left(V^{2}\right)^{*}=W^{*} \text {. }
$$

Further, using that $V^{2}=W$ we find $V W V^{*}=V V^{2} V^{*}=V^{2}=W$

Before we use this assertion about the structure of algebraic conjugations we construct for a large class of non type I factors $\mathcal{M}_{0}$ algebraic conjugations which are not modular conjugations (shortly we call such algebraic conjugations purely algebraic conjugations). The construction uses the flip automorphism and the fact that "many" factors $\mathcal{M}_{0}$ are tensor squares, $\mathcal{M}_{0} \cong \mathcal{N} \bar{\otimes} \mathcal{N}$.

Theorem 4.5. Let $\mathcal{M}_{0}$ be a separable von Neumann factor with a cyclic and sep. arating vector $u_{0}$. Suppose $\mathcal{M}_{0}$ is isomorphic to $\mathcal{N} \bar{\otimes} \mathcal{N}$ where $\mathcal{N}$ is a von Neumann factor and $\mathcal{M}_{0}$ is not a type $I$ factor. Then there is an algebraic conjugation for $\mathcal{M}_{0}$ which is not a modular conjugation.

Proof. 1. Since $\mathcal{M}_{0}$ is separable the factor $\mathcal{N}$ is also separable. Thus $\mathcal{N}$ is isomorphic to a von Neumann factor $\mathcal{L}$ on a separable Hilbert space and $\mathcal{L}$ has a cyclic and separating vector $v_{o}$ (see [3: Proposition 2.5.6]). This implies that $\mathcal{L} \bar{\otimes} \mathcal{L}$ is a von Neumann factor with the cyclic and separating vector $v:=v_{0} \otimes v_{o}$ (see [9]). Further, $\mathcal{M}_{0}$ is isomorphic to $\mathcal{L} \bar{\otimes} \mathcal{L}$. Since both von Neumann factors have a cyclic and separating vector they are spatially isomorphic. Thus, without loss of generality, we can assume in the following that $\mathcal{M}_{o}=\mathcal{N} \otimes \mathcal{N}$ on $\mathcal{H}=\mathcal{H}_{0} \otimes \mathcal{H}_{0}$ and $\mathcal{M}_{0}$ has a cyclic and separating vector $u=u_{o} \otimes u_{o}$.

2. We consider the flip automorphism $\alpha$ on $\mathcal{N} \bar{\otimes} \mathcal{N}$ given by $\alpha(X \otimes Y)=Y \otimes$ $X(X, Y \in \mathcal{N})$. We obtain

$$
(u, \alpha(X \otimes Y) u)=(u, Y \otimes X u)=\left(u_{0}, Y u_{0}\right)\left(u_{0}, X u_{o}\right)=(u, X \otimes Y u) .
$$

This gives that $\alpha$ leaves invariant the vector state $(u, \cdot u)$. Thus, for $\alpha$ there exist a unitary operator $V_{\alpha}$ such that ad $V_{\alpha}=\alpha, V_{\alpha} u=u$, and $V_{\alpha}$ commutes with the modular objects $\left(\Delta_{o}, J_{o}\right)$ for $\left(\mathcal{M}_{o}, u\right)$ (see, e.g., [3]). Since $\mathcal{M}_{0}$ is not of type I we have that $\alpha$ (the flip) is an outer automorphism (see [11]).

3. Next we show that $V_{\alpha}^{2}=1$. Since $\alpha^{2}$ is the identity automorphism we find that $\alpha^{2}(M)=V_{\alpha}^{2} M\left(V_{\alpha}^{*}\right)^{2}=M\left(M \in \mathcal{M}_{o}\right)$ and therefore $Y:=V_{\alpha}^{2} \in \mathcal{M}_{o}^{\prime}$. This implies, with $V_{\alpha} u=u, M u=M V_{\alpha}^{2} u=V_{\alpha}^{2} M u\left(M \in \mathcal{M}_{0}\right)$. Since $u$ is cyclic for $\mathcal{M}_{o}$ we get that $V_{\alpha}^{2}=1$, thus $V_{\alpha}=V_{\alpha}^{*}$. 
4. Now we define an antiunitary operator $J=V_{\alpha} J_{o}$. Since $V_{\alpha}$ commutes with the modular conjugation $J_{o}$ (see Step 2) we have $J^{2}=V_{\alpha} J_{o} V_{\alpha} J_{o}=V_{\alpha}^{2} J_{o} J_{o}=1$, i.e. $J$ is a conjugation. Further,

$$
J \mathcal{M}_{o} J=V_{\alpha} J_{0} \mathcal{M}_{0} V_{\alpha} J_{o}=J_{0} V_{\alpha} \mathcal{M}_{o} V_{\alpha} J_{o}=J_{o} \alpha\left(\mathcal{M}_{0}\right) J_{o}=J_{o} \mathcal{M}_{0} J_{o}=\mathcal{M}_{o}^{\prime}
$$

i.e. $J$ is an algebraic conjugation for $\mathcal{M}_{0}$. Since $V_{\alpha}=J J_{0}$ implements an outer automorphism of $\mathcal{M}_{0}$ we obtain from Proposition 4.2 that $J$ is an algebraic conjugation which is not a modular conjugation

The next question is which von Neumann factors are tensor squares. First, we note that not all von Neumann factors have such a structure. For example, finite von Neumann factors of type $I_{n}$ where $n$ is not a square of a natural number are not tensor squares. Second, for some non-hyperfinite type $I I_{1}$ factors it is not known if they have outer automorphisms and therefore it is not clear if they are tensor squares (tensor squares of non type I factors have an outer automorphism, the flip automorphism, [1i]). Third, there are hyperfinite $I I \dot{I}_{0}$ factors (even ITPFI factors) which are not tensor squares (see [7]).

The following proposition presents a class of von Neumann factors which are tensor squares. It follows directly from well known results in the literature.

Proposition 4.6. Let $\mathcal{M}_{0}$ be a hyperfinite factor. Then:

(i) If $\mathcal{M}_{0}$ is neither of type $I$ nor of type $I I I_{0}$, then $\mathcal{M}_{0}$ is a tensor square, i.e. $\mathcal{M}_{o} \cong \mathcal{N} \bar{\otimes} \mathcal{N}$ where $\mathcal{N}$ is a hyperfinite factor.

(ii) If $\mathcal{M}_{o}$ is a type $I I I_{o}$ factor whose flow of weights has pure point spectrum, then $\mathcal{M}_{0} \cong \mathcal{N} \bar{\otimes} \mathcal{N}$ where $\mathcal{N}$ is a hyperfinite factor.

Proof. 1. First we note that the tensor square $\mathcal{N} \bar{\otimes} \mathcal{N}$ of a hyperfinite factor $\mathcal{N}$ is again a hyperfinite factor. This follows from the fact that such factors are injective (we consider only separable von Neumann factors where injective is the same as hyperfinite) and injectivity is stable under tensor products (see, e.g., [5]), and from the fact that the tensor product of factors is again a factor (see [9]).

2. If $\mathcal{N}$ is a hyperfinite factor of type $I I_{1}\left(I I_{\infty}\right)$, then $\mathcal{N} \bar{\otimes} \mathcal{N}$ is again a factor of type $I I_{1}\left(I I_{\infty}\right)$ (see [9]), and it is hyperfinite because of Step 1. Since these factors are üique (see, e.g., [5]), we get that the hyperfinite factors of type $I I_{1}$ and of type $I I_{\infty}$ are tensor squares.

3. There exists a hyperfinite type $I I I_{0}$ factor $\mathcal{N}$ such that $\mathcal{M}=\mathcal{N} \bar{\otimes} \mathcal{N}$ is a hyperfinite type $I I I_{1}$ factor (a hyperfinite type $I I I_{\lambda}$ factor, for each $\lambda \in(0,1)$ ) (see [6]). Since the hyperfinite factors of type $I I I_{1}$ and of type $I I I_{\lambda}, \lambda \in(0,1)$ are unique (see [5]), we obtain that they are tensor squares.

4. If $\mathcal{M}_{0}$ is a hyperfinite type $I I I_{0}$ factor whose flow of weights has pure point spectrum, then there is a hyperfinite type $I I I_{0}$ factor $\mathcal{N}$ with $\mathcal{M}_{0} \cong \mathcal{N} \bar{\otimes} \mathcal{N}$ (see [6])

Remark 4.7. 1. From Proposition 4.6 and Theorem 4.5 we get that all hyperfinite factors of type $I I, I I I_{\lambda}, I I I_{1}$, and $I I I_{o}$ (whose flow of weights has pure point spectrum) have purely algebraic conjugations. 
2. For the hyperfinite factors described in item 1 we have that they are even tensor squares of themselves, i.e. $\mathcal{M} \cong \mathcal{M} \bar{\otimes} \mathcal{M}$ (see $[6,10])$.

For factors which are not hyperfinite or do not belong to the set of hyperfinite type $I I I_{\text {o }}$ factors described in Proposition 4.6 the existence of purely algebraic conjugations is not clear.

At the end of this section we shortly discuss the question how "large" the set of purely algebraic conjugations is.

From Proposition 4.2 we see that the set of algebraic conjugations for a von Neumann factor $\mathcal{M}_{0}$ is given by

$$
\left\{J=V \cdot J_{o}: \text { ad } V \in \text { aut } \mathcal{M}_{o} \text { and } V J_{o}=J_{o} V^{*}\right\}
$$

where $J_{o}$ is a fixed modular conjugation of $\mathcal{M}_{0}$. Thus the set of algebraic conjugations is determined by the set of automorphisms ad $V$. Now the set of automorphisms of $\mathcal{M}_{0}$ can be classified with the help of the outer conjugacy relation. Two automorphisms $\alpha$ and $\beta$ are said to be outer conjugate if there is a $\theta \in$ aut $\mathcal{M}_{0}$ such that $\beta=\theta \cdot \alpha \cdot \theta^{-1}$ modulo int $\mathcal{M}_{0}$. In our case $(\operatorname{ad} V)^{p}=\operatorname{ad} W \in \operatorname{int} \mathcal{M}_{0}$ and $\operatorname{ad} V(W)=\gamma W$ with $p=2$ and $\gamma=1$ (see Proposition 4.4). This means the automorphisms ad $V$ defining the purely algebraic conjugations have the outer conjugacy class invariants $p(\operatorname{ad} V)=2$ and $\gamma(\operatorname{ad} V)=1$ ) (for details about outer conjugacy, see [5: Section V.6]). Thus we get the following result.

Proposition 4.8. Let $\mathcal{M}_{0}$ be a hyperfinite factor of type II with a cyclic and sepa. rating vector $u_{0}$. Let $J_{0}$ be the modular conjugation for $\left(\mathcal{M}_{0}, u_{0}\right)$. Further, let $J_{1}=V_{1} \cdot J_{0}$ and $J_{2}=V_{2} \cdot J_{0}$ be two purely algebraic conjugations for $\mathcal{M}_{0}$. Then ad $V_{1}$ and ad $V_{2}$ are outer conjugate.

Proof. From the considerations before we know that ad $V_{1}$ and ad $V_{2}$ have the same invariants $p=2$ and $\gamma=1$. From [5: Section V.6/Theorems 14 and 16] it follows that then ad $V_{1}$ and ad $V_{2}$ are outer conjugate

Remark 4.9. 1. From this proposition and Remark 4.7 we see that for hyperfinite type II factors there exists only one purely algebraic conjugation up to outer conjugacy. One can partly extend this result to such hyperfinite type III factors as described in Remark 4.7 with the help of the classification of the automorphisms of these factors (see [16]). We omit the details.

2. Naturally, if an outer automorphism ad $V$ of $\mathcal{M}_{0}$ satisfies $p(\operatorname{ad} V)=2$ and $\gamma(\operatorname{ad} V)=1$, then $J:=V \cdot J_{0}$ is in generally not an algebraic conjugation for $\mathcal{M}_{0}$. The relation $J^{2}=1$ does not follow from these assumptions.

Acknowledgments. We thank one of the referees for the suggestion to separate a part of the proof of Proposition 2.2 as a lemma (Lemma 2.1). This paper is partly based on the Diploma thesis of the first author. 


\section{Appendix}

In this appendix we prove two simple but useful lemmas.

Lemma 5.1. Suppose $U=U_{1} \cdot U_{2}$ with elements $U_{1} \in \mathcal{M}$ and $U_{2} \in \mathcal{M}^{\prime}$ which are bounded invertible. Then this decomposition of $U$ in bounded invertible elements from $\mathcal{M}$ and $\mathcal{M}^{\prime}$ is unique up to a bounded invertible element from the center of $\mathcal{M}$. This means if there is another such decomposition $U=V_{1} \cdot V_{2}$ with $V_{1} \in \mathcal{M}$ and $V_{2} \in \mathcal{M}^{\prime}$, then $U_{1}=G \cdot V_{1}$ and $U_{2}=G^{-1} \cdot V_{2}$ with $G \in \mathcal{Z}(\mathcal{M})$.

Proof. If $U=U_{1} \cdot U_{2}=V_{1} \cdot V_{2}$ with $U_{1}, V_{1} \in \mathcal{M}$ and $U_{2}, V_{2} \in \mathcal{M}^{\prime}$, then we get that $G:=V_{1}^{-1} U_{1}=V_{2} U_{2}^{-1}$ is from $\mathcal{M} \cap \mathcal{M}^{\prime}$. Clearly, $G$ has an inverse. This proves the assertion

Lemma 5.2. Let $H$ be a selfadjoint operator on a separable Hilbert space $\mathcal{H}$. Suppose $H$ has the eigenvalue 1 . Let $u_{1}, u_{2}$ be two unit eigenvectors of $H$ to the eigenvalue 1. Suppose there is an antiunitary operator $J$ with $J^{2}=1, J u_{1}=u_{1}$, and $J u_{2}=u_{2}$. Then there is a unitary operator $V_{1}$ on $\mathcal{H}$ with the properties $V_{1} H=H V_{1}, V_{1} J=J V_{1}$, and $V_{1} u_{1}=u_{2}$.

Proof. 1. Assume $u_{1}=e^{i a} u_{2}$. Then we get

$$
u_{1}=J u_{1}=J e^{i a} u_{2}=e^{-i a} J u_{2}=e^{-i a} u_{2}=e^{-i 2 a} u_{1} .
$$

Thus, $e^{i a}$ is 1 or -1 . So we can choose $V_{1}$ as 1 or -1 .

2. Next assume that $u_{1}, u_{2}$ generate a two-dimensional subspace $\mathcal{H}_{0}$ of $\mathcal{H}$. We can choose orthonormal bases $\left\{u_{1}, u_{1}^{\prime}\right\}$ and $\left\{u_{2}, u_{2}^{\prime}\right\}$ in $\mathcal{H}_{0}$ such that $J u_{j}^{\prime}=u_{j}^{\prime}(j=1,2)$. Namely, suppose that $\left\{u_{1}, v_{1}\right\}$ is an orthonormal basis in $\mathcal{H}_{0}$ such that $J v_{1} \neq v_{1}$. We have $\left(J v_{1}, u_{1}\right)=\left(J u_{1}, v_{1}\right)=\left(u_{1}, v_{1}\right)=0$. Further, $J \mathcal{H}_{0}=\mathcal{H}_{0}$. This gives that $\left\{u_{1}, J v_{1}\right\}$ is again an orthonormal basis in $\mathcal{H}_{0}$ like $\left\{u_{1}, v_{1}\right\}$. Thus $J v_{1}=e^{i c} v_{1}$.

Now we set $u_{1}^{\prime}=e^{i c / 2} v_{1}$. Then $J u_{1}^{\prime}=J e^{i \frac{c}{2}} v_{1}=e^{-i \frac{c}{2}} J v_{1}=e^{i \frac{c}{2}} v_{1}=u_{1}^{\prime}$. Clearly, $u_{1}^{\prime}$ is from $\mathcal{H}_{o}$ and is orthogonal to $u_{1}$. This shows $\left\{u_{1}, u_{1}^{\prime}\right\}$ has the right invariance under $J$. The same can be done with $\left\{u_{2}, u_{2}^{\prime}\right\}$. So our assumption on the bases can be fulfilled. Next we define $V_{o}$ as a partial isometry from $\mathcal{H}_{o}$ onto $\mathcal{H}_{o}$ with $V_{o} u_{1}=u_{2}$ and $V_{o} u_{1}^{\prime}=u_{2}^{\prime}$.

3. We make the ansatz $V_{1}:=\left(1-P_{0}\right)+V_{o}$ where $P_{0}$ is the orthoprojection onto $\mathcal{H}_{0}$. Clearly, $P_{o}$ commutes with $H$ and $V_{o}$ commutes with $H$. Thus $V_{1} H=H V_{1}$. Further, we get $V_{1} u_{1}=V_{o} u_{1}=u_{2}$.

4. It remains to show $V_{1} J=J V_{1}$. First from $J u_{j}=u_{j}$ and $J^{2}=1$ it follows $J P_{\mathrm{o}}=P_{\mathrm{o}} J$. Second, we have for $u \in \mathcal{H}_{o}$

$$
\left.\begin{array}{l}
J V_{o} u=J V_{o}\left(a_{1} u_{1}+a_{2} u_{1}^{\prime}\right)=J\left(a_{1} u_{2}+a_{2} u_{2}^{\prime}\right)=\bar{a}_{1} u_{2}+\bar{a}_{2} u_{2}^{\prime} \\
V_{o} J u=V_{o} J\left(a_{1} u_{1}+a_{2} u_{1}^{\prime}\right)=V_{o}\left(\bar{a}_{1} u_{1}+\bar{a}_{2} u_{1}^{\prime}\right)=\bar{a}_{1} u_{2}+\bar{a}_{2} u_{2}^{\prime} .
\end{array}\right\}
$$

This implies $J V_{o}=V_{o} J$. Thus $J V_{1}=V_{1} J$. This concludes the proof 


\section{References}

[1] Baumgärtel, H. and M. Wollenberg: Mathematical Scattering Theory. Berlin: AkademieVerlag 1983.

[2] Baumgärtel, H. and M. Wollenberg: Causal Nets of Operator Algebras. Berlin: A kademieVerlag 1992.

[3] Bratteli, O. and D. W. Robinson: Operator Algebras and Quantum Statistical Mechanics, Vol. I. New York: Springer 1979.

[4] Borchers, H. J.: A noncommuting realization of Minkowski space. In: Quantum and Non-Commutative Analysis (ed.: H. Araki et al.). Dordrecht: Kluwer 1993, pp. $11-30$.

[5] Connes, A.: Noncommutative Geometry. New York: Academic Press 1994.

[6] Giordano, T. and G. Skandalis: Krieger factors isomorphic to their tensor square and pure point spectrum flows. J. Funct. Anal. 64 (1985), $209-226$.

[7] Giordano, T., Skandalis, G. and E. J. Woods: On the computation of invariants for ITPFI factors. J. Oper. Theory 15 (1986), 83 - 107.

[8] Haag, R.: Local Quantum Physics, 2nd ed. Berlin: Springer 1996.

[9] Kadison, R. V. and J. R. Ringrose: Fundamentals of the Theory of Operator Algebras, Vol II. New York: Academic Press 1986.

[10] Leitz, M.: Über Modularkonjugationen von von-Neumann-Algebren. Diploma Thesis. Leipzig: Universität 1997.

[11] Sakai, S.: Automorphisms and tensor products of operator algebras. Amer. J. Math. 97 (1976), $889-896$.

[12] Schroer, B.: Motivations and physical aims of algebraic quantum field theory. Ann. Physics 255 (1997), $270-304$.

[13] Stratila, S.: Modulor Theory in Operator Algebras. Bucuresti: Editura Academiei, and Tunbridge Wells: Abacus Press 1981.

[14] Stratila, S. and L. Zsido: Lectures on von Neumann algebras. Tunbridge Wells: Abacus Press 1979.

[15] Takesaki, M.: The Theory of Operator Algebras. New York: Springer 1979.

[16] Takesaki, M.: The structure of the automorphism group of an AFD factor. Progr. Math. 92 (1990), 19 - 44.

[17] Wollenberg, M.: An inverse problem in modular theory, Part I. Preprint. Leipzig: Univ., Preprint NTZ 32/1997.

Received 14.04.1999 